\title{
Clima motivacional em jogadores de uma equipa de andebol
}

\author{
Motivational climate in a team handball players
}

\author{
J. Vasconcelos-Raposo, J.M. Moreira, C.M. Teixeira
}

ARTIGO ORIGINAL | ORIGINAL ARTICLE

\begin{abstract}
A orientação motivacional de um atleta visa a melhoria das suas capacidades (tarefa) e a obtenção de um bom resultado numa dada competição (ego). Desta forma, objetivou-se comparar andebolistas de um clube português de diferentes escalões competitivos, anos de prática da modalidade, tempo de presença no clube e posição competitiva ao nível das suas orientações motivacionais. A amostra contou com 57 atletas do sexo masculino nascidos entre 1977 e 1997. Foi utilizado o Questionário sobre a Orientação para a Tarefa e para o Ego no Desporto (TEOSQp) de Fernandes e Vasconcelos-Raposo (2010). Os resultados revelam que os andebolistas deste estudo têm uma forte orientação para a tarefa, apresentando uma média de 4.03 ( \pm .37), superior à média da orientação para o ego (3.78 \pm .49$)$, o que vai de encontro aos valores obtidos noutros estudos que consolidaram a proposta teórica. Averiguou-se ainda que existem diferenças significativas entre os escalões na orientação para a tarefa e que os atletas sénior apresentam as médias mais altas na orientação para a tarefa e as mais baixas na orientação para o ego. Os anos de prática da modalidade, o tempo de presença no clube e a posição competitiva não parecem influenciar, significativamente, a orientação motivacional dos andebolistas. Palavras-chave: orientação para o ego, tarefa, andebol
\end{abstract}

ABSTRACT

The motivational orientation of an athlete aims to improve their skills (task) and getting a good result in a given competition (ego). Thus, the purpose of the present study was to compare players of one Portuguese team handball club. The independent variables were: competitive levels, years as competitors, time presence as player in the club and competitive position. The dependent variables were motivational orientations: task and ego. The sample consisted of 57 male athletes borne between 1977 and 1997. The Task and Ego Orientation Questionnaire in Sport (TEOSQp), validated by Fernandes and Vasconcelos-Raposo (2010) was used. Results revealed that the handball players in the club studied present high scores on both task and ego orientation with a higher score at the level of task orientation. The present results are not in accordance with previous studies. However they do not contradict consolidated theoretical proposal. Senior athletes presented higher scores in task orientation and lower on ego. Comparisons by years in competition, time presence in the club and competitive position in the do influence significantly the motivational orientation among this club team handball players. Keywords: task and ego orientation; handball

Submetido: 16.12.2012 | Aceite: 24.05.2013

José Vasconcelos-Raposo, Joana Mendes Moreira, Carla Maria Teixeira. Universidade de Trás-os-Montes e Alto Douro, Vila Real, Portugal.

Endereço para correspondência: José Vasconcelos-Raposo, Universidade de Trás-os-Montes e Alto Douro. Rua Dr. Manuel Cardona, 5000-558 Vila Real, Portugal.

E-mail: jvraposo@utad.pt 
A motivação representa forças externas e internas que produzem a iniciação, direção, intensidade e persistência de um comportamento direcionado a um objetivo específico e é uma das variáveis cognitivas determinantes na adoção e manutenção de hábitos desportivos (Buckworth \& Dishman, 2007). Sem a motivação apropriada é difícil que os atletas se dediquem à prática desportiva com o sacrifício e perseverança que é exigido ao mais alto nível de rendimento desportivo.

Para Vasconcelos-Raposo e Mahl (2005) a motivação pode ser definida como a direção e intensidade de um esforço. Se um indivíduo procura aproximar-se ou é atraído para determinadas situações, estamos perante a direção do esforço. A quantidade de esforço que um atleta coloca numa determinada situação refere-se à intensidade do esforço (Weinberg \& Gould, 2007).

A psicologia da motivação tem como objetivo responder a questões gerais como: por que razão é que alguns indivíduos participam em desportos e outros não? Por que é que uns atletas praticam futebol e outros atletismo ou esqui? Quais os fatores que são importantes para manter alguém otimamente motivado num desporto e como manter esse nível ótimo de motivação? Ou por outro lado, por que é que alguns atletas abandonam antecipadamente a prática desportiva?

Parece existir consenso quando atletas e treinadores afirmam que um atleta com elevados níveis de motivação utiliza a sua energia para estabelecer objetivos e para cumpri-los, emprega vontade de resolver os problemas, evidencia elevados níveis de atenção e concentração e demonstra entusiasmo e gosto pela participação desportiva. Por estas razões é da maior importância que a motivação e os processos associados a esta sejam objecto de permanente preocupação por parte das equipas técnicas.

A motivação para a realização afeta o desempenho e a participação no que diz respeito à prática. Relaciona-se com os esforços de um indivíduo em dominar uma tarefa, atingir os seus limites, superar obstáculos, ter um melhor desempenho que os outros e ter orgulho do seu talento (Ferreira, Costa, Penna, Samulsky, \& Moraes, 2012).

Diferenciam-se dois tipos de orientações motivacionais: a orientação para a tarefa e a orientação para o ego. Orientação para a tarefa (ou mestria) é a preocupação do atleta no desenvolvimento da sua competência e das suas habilidades na melhoria de uma tarefa (Vasconcelos-Raposo \& Mahl, 2005). Um indivíduo com esta orientação motivacional sentir-se-á bem-sucedido quando atingir o desenvolvimento de mestria e melhorar as suas próprias capacidades. Arriscamos afirmar que o atleta está motivado a melhorar os seus níveis de desempenho anteriores, tendo em vista a autossuperação.

Um indivíduo orientado para o ego não objetiva superar-se a si mesmo, mas sim superar os outros, a sua perceção de sucesso baseia-se na comparação com os colegas, tendo necessidade de se sentir superior a eles. Vasconcelos-Raposo e Mahl (2005) defendem que o critério de alta perceção de habilidade dos atletas orientados para o ego (resultado) depende do alcance de resultados tão bons ou melhor do que os outros e de preferência num menor esforço possível.

Em idades inferiores, a própria habilidade é vista pelo atleta como melhor ou pior tendo em conta o desempenho realizado anteriormente por ele mesmo, em idades mais avançadas há já uma tendência de comparar a sua habilidade com a habilidade dos outros (Cox, 2007).

Silva (2006) encontrou elevada orientação para a tarefa nos atletas de escalões competitivos superiores. Contudo, num estudo de Coroa (2009) este verificou que atletas muito novos, com idades compreendidas entre os 8 e os 11 anos, também se orientam preferencialmente para a tarefa. Libório (2007) encontrou valores superiores relativamente à orientação para o ego nos escalões inferiores. 
Rodrigues, Lázaro, Fernandes e Vasconcelos-Raposo (2009) verificaram que a orientação para a tarefa é superior nos atletas com mais anos de experiência. Nesse mesmo estudo não se encontraram diferenças significativas entre os anos de prática da modalidade e a orientação para o ego. Num estudo de Vasconcelos-Raposo e Mahl (2005) verificou-se que a posição competitiva não evidenciou diferenças significativas relativamente à orientação cognitiva adotada.

Face aos resultados constatados na literatura optamos por assumir como objectivos específicos para o presente trabalho fazer as seguintes comparações em função das orientações motivacionais: 1- escalão competitivo; 2- anos de prática; e 3- anos de presença na eauipa; 4- posição competitiva.

\section{MÉTODO}

\section{Amostra}

A amostra é constituída por 57 atletas nascidos entre 1977 e 1997 do sexo masculino de diferentes escalões competitivos, 14 (24.6\%) iniciados nascidos em 1996 e 1997, 21 (36.8\%) juvenis nascidos entre 1993 e 1995, 8 (14.0\%) juniores nascidos entre 1990 e 1992 e 14 (24.6\%) seniores nascidos entre 1977 e 1992. Os anos de prática da modalidade variaram entre 1 e 22 anos, os atletas foram divididos em 4 grupos, 18 (31.6\%) têm 3 ou menos anos de prática de andebol, entre 4 e 8 anos eram 17 (29.8\%), entre 9 e 13 anos eram 14 (24.6\%) e com mais de 14 anos de prática de andebol eram 8 (14.0\%). Os anos de presença no clube variaram entre 1 e 13 anos, foram divididos em 4 grupos, os que se encontravam há um ano ou menos no clube eram 17 (29.8\%), com 2 a 4 anos eram 27 (47.4\%), com 5 a 7 anos eram $5(8.8 \%)$ e $8(14.0 \%)$ estavam há 8 anos ou mais no clube. Relativamente à posição competitiva eram 9 (15.8\%) guarda-redes, $9(15.8 \%)$ centrais, $12(21.1 \%)$ laterais, 11 (19.3\%) pontas, 10 (17.5\%) pivots e $6(10.5 \%)$ universais.

\section{Instrumentos}

O Questionário de Orientação para a Tarefa e para o Ego no Desporto (TEOSQp) visa saber a opinião do atleta acerca do significado do sucesso no contexto desportivo. A versão utilizada foi desenvolvida por Fernandes e Vasconcelos-Raposo (2010) e é constituída por 13 itens que se encontram agrupados em dois fatores de orientação cognitiva, 6 de orientação para o ego e 7 de orientação para a tarefa. Os 13 itens foram respondidos numa escala tipo Likert de 5 pontos (variando entre $1=$ discordo completamente e $5=$ concordo completamente).

\section{Procedimentos}

Os questionários foram entregues a todos os atletas pertencentes à modalidade de um clube profissional de andebol português. Para o tratamento estatístico dos dados recorreu-se ao programa SPSS 20 (Statistical Package for the Social Sciences). Através do cálculo da média, desvio padrão, valor máximo e mínimo realizou-se a análise descritiva dos dados. O estudo da normalidade das distribuições foi concretizado através da análise das medidas de simetria (Skewness) e achatamento (Kurtosis). A homogeneidade das variâncias foi analisada através do teste de Levene. O efeito das variáveis independentes no conjunto das dimensões abrangidas pelos instrumentos utilizados foi estudado através da análise de variância multivariada (MANOVA a um fator). Foram utilizados os testes Post-Hoc para comparação entre grupos e identificação de possíveis diferenças significativas. Estabeleceu-se um nível de significância de $5 \%(p \leq .05)$ em toda a análise dos dados.

\section{RESULTADOS}

No quadro 1 são apresentados a média, desvio padrão, os valores mínimo e máximo, o Skewness e Kurtosis das orientações cognitivas da população em estudo.

Uma vez que o TEOSQp contempla uma escala tipo Likert de 5 pontos, pode-se afirmar que os andebolistas participantes nesta inves- 
Quadro 1

análise descritiva das orientações cognitivas

\begin{tabular}{|c|c|c|c|c|c|c|c|c|}
\hline & \multirow{2}{*}{ Mínimo } & \multirow{2}{*}{ Máximo } & \multirow{2}{*}{ Média } & \multirow{2}{*}{ Desvio Padrão } & \multicolumn{2}{|c|}{ Skewness } & \multicolumn{2}{|c|}{ Kurtosis } \\
\hline & & & & & Estatística & Erro & Estatística & Erro \\
\hline Orie & 3.00 & 4.8 & 4.03 & 0.3 & -.10 & .32 & -.07 & .62 \\
\hline Orientação Ego & 2.50 & 4.67 & 3.78 & 0.49 & -.40 & .32 & .15 & .62 \\
\hline
\end{tabular}

tigação têm uma forte orientação para a tarefa, apresentando uma média de 4.03 ( \pm .37). A orientação para o ego tem uma média inferior à orientação para a tarefa $(3.78 \pm .49)$. Analisando o Skewness e Kurtosis verifica-se a normalidade das distribuições.

\section{Escalão competitivo}

Quanto à comparação entre escalões no que toca às orientações cognitivas, a análise de variância multivariada (Manova a um fator) revelou existir um efeito diferenciador significativo $\left[\lambda\right.$ de Wilks $=.788, \mathrm{~F}_{(3,53)}=2.193, p=$ .049 e $\left.\eta_{p}^{2}=.112\right]$. Apesar de apenas a orientação para a tarefa $(p=.007)$ mostrar diferenças significativas, ambas as dimensões do TEOSQp parecem evidenciar algumas relações causa-efeito. Foi obtido efeito forte, sugerindo que $21 \%$ da variação na combinação linear da orientação para a tarefa pode ser atribuído ao escalão competitivo. Foram obtidos efeitos médios, sugerindo que $11 \%$ da variação na combinação linear da orientação para o ego pode ser atribuído ao escalão competitivo.

Os seniores evidenciam médias superiores na orientação para a tarefa e os iniciados evidenciam uma média mais alta na orientação para o ego. Os juvenis são aqueles que apresentam médias mais baixas nas orientações cognitivas, havendo diferenças significativas entre este grupo e o grupo de seniores, especialmente na orientação para a tarefa em que o escalão superior apresenta médias mais altas.

\section{Anos de prática da modalidade}

Para diferenciar a experiência competitiva quanto às dimensões do TEOSQp, procedeu-se a uma Manova (a um fator) que denunciou não existir um efeito diferenciador significativo por parte da variável anos de prática da modalidade $\left[\lambda\right.$ de Wilks $=.859, \mathrm{~F}_{(3,53)}=1.368, p=.234$ e $\left.\eta_{p}^{2}=.073\right]$. O teste de Levene demonstrou que a igualdade de variâncias entre os grupos pode ser assumida em ambas as orientações cognitivas. Apesar de nenhuma das orientações cognitivas mostrar diferenças significativas, ambas parecem evidenciar algumas relações causa-efeito. Foram obtidos efeitos médios, sugerindo que $12 \%$ (orientação para a tarefa) e $6 \%$ (orientação para o ego) das variações na combinação linear das variáveis dependentes podem ser atribuídos aos anos de prática da modalidade.

De um modo geral não houve um aumento

Quadro 2

Comparação e diferenciação das orientações cognitivas em função do escalão

\begin{tabular}{ccccccc}
\hline & $\begin{array}{c}\text { Iniciados (I) } \\
(n=14)\end{array}$ & $\begin{array}{c}\text { Juvenis (J1) } \\
(n=21)\end{array}$ & $\begin{array}{c}\text { Juniores (J2) } \\
(n=8)\end{array}$ & $\begin{array}{c}\text { Seniores } \\
(n=14)\end{array}$ & $\eta_{p}^{2}$ & $\begin{array}{c}\neq \text { significativas } \\
\text { entre grupos } \\
(\text { Post-Hoc) }\end{array}$ \\
\hline M (DP) & $\mathrm{M}(\mathrm{DP})$ & $\mathrm{M}(\mathrm{DP})$ & $\mathrm{M}(\mathrm{DP})$ & $\mathrm{S})$ \\
\hline Orientação Tarefa & $4.17( \pm .45)$ & $3.86( \pm .29)$ & $3.89( \pm .20)$ & $4.22( \pm .35)$ & .205 & $\mathrm{~S}>\mathrm{J} 1$ \\
Orientação Ego & $3.95( \pm .50)$ & $3.60( \pm .42)$ & $3.69( \pm .23)$ & $3.93( \pm .63)$ & .106 & -- \\
\hline
\end{tabular}

Quadro 3

Comparação e diferenciação das orientações cognitivas em função dos anos de prática da modalidade

\begin{tabular}{cccccc}
\hline & $\leq 3(\mathrm{E} 1)$ & ] $3-8](\mathrm{E} 2)$ & ] $8-13](\mathrm{E} 3)$ & $>13(\mathrm{E} 4)$ & \\
$(n=18)$ & $(n=17)$ & $(n=14)$ & $(n=8)$ & $\eta_{p}^{2}$ \\
& $\mathrm{M}(\mathrm{DP})$ & $\mathrm{M}(\mathrm{DP})$ & $\mathrm{M}(\mathrm{DP})$ & $\mathrm{M}(\mathrm{DP})$ & \\
\hline Orientação Tarefa & $4.09( \pm .44)$ & $3.89( \pm .31)$ & $3.98( \pm .26)$ & $4.29( \pm .40)$ & .122 \\
Orientação Ego & $3.80( \pm .52)$ & $3.62( \pm .43)$ & $3.83( \pm .37)$ & $4.00( \pm .71)$ & .064 \\
\hline
\end{tabular}


progressivo das médias de ambas as orientações cognitivas com o aumento dos anos de prática da modalidade. Contudo, o grupo dos que praticam a modalidade há mais tempo evidenciou médias superiores aos restantes grupos tanto na orientação para a tarefa como na orientação para o ego. O grupo 2 (4-8) evidenciou médias mais baixas para ambas as orientações cognitivas.

\section{Tempo de presença no clube}

Quanto à comparação entre grupos no que toca às orientações cognitivas, a Manova revelou não existir um efeito diferenciador significativo $\left[\lambda\right.$ de Wilks $=.828, \mathrm{~F}_{(3,53)}=1.713$, $\mathrm{p}=.125$ e $\left.\eta_{p}^{2}=.090\right]$. Apesar de apenas a orientação para a tarefa $(p=.03)$ mostrar diferenças significativas, ambas as dimensões do TEOSQp parecem evidenciar algumas relações causa-efeito. Foi obtido efeito forte, sugerindo que $15 \%$ da variação na combinação linear da orientação para a tarefa pode ser atribuído ao tempo de presença no clube. Foi obtido efeito médio, sugerindo que $7 \%$ da variação na combinação linear da orientação para o ego pode ser atribuído ao tempo de presença no clube.

De uma forma generalizada não se verificou um aumento ou diminuição graduais nas orientações cognitivas com o aumento do tempo de presença no clube. O grupo2 (2-4 anos) mostrou média mais alta na orientação para a tarefa; quanto à orientação para o ego, aqueles que se encontram há mais tempo no clube apresentaram média mais alta do que os restantes grupos. O grupo 3 (5-7) evidenciou médias mais baixas para ambas as orientações cognitivas.

\section{Posição competitiva}

Com o objetivo de comparar as diferentes posições competitivas, efetuou-se uma Manova (a um fator) que revelou não existir um efeito diferenciador significativo por parte da variável posição competitiva $\left[\lambda\right.$ de Wilks $=.832, \mathrm{~F}_{(5,51)}$ $=.965, p=.478$ e $\left.\eta_{p}^{2}=.088\right]$. Apesar de nenhuma das orientação cognitivas mostrar diferenças significativas, ambas as dimensões do TEOSQp parecem evidenciar algumas relações causa-efeito. Foram obtidos efeitos médios, sugerindo que $9 \%$ (orientação para o ego) e $6 \%$ (orientação para a tarefa) das variações na combinação linear das variáveis dependentes podem ser atribuídos à posição competitiva.

De uma forma geral não existem diferenças evidentes entre as várias posições competitivas no que toca a ambas às orientações cognitivas. Os jogadores universais são aqueles que apresentam uma média superior na orientação para a tarefa e inferior na orientação para o ego. Os pontas são os jogadores que apresentam uma média superior na orientação para o ego e os centrais os que têm média inferior na orientação para a tarefa.

Quadro 4

Comparação e diferenciação das orientações cognitivas em função do tempo de presença no clube

\begin{tabular}{cccccc}
\hline & GR $1: \leq 1$ & GR 2: ]1-4] & GR 3: ]5-7] & GR 4: $>7$ & \\
$(n=17)$ & $(n=27)$ & $(n=5)$ & $(n=8)$ & $\eta_{p}^{2}$ \\
& $\mathrm{M}(\mathrm{DP})$ & $\mathrm{M}(\mathrm{DP})$ & $\mathrm{M}(\mathrm{DP})$ & $\mathrm{M}(\mathrm{DP})$ & .154 \\
\hline Orientação Tarefa & $3.87( \pm .36)$ & $4.15( \pm .32)$ & $3.77( \pm .30)$ & $4.13( \pm .45)$ & .069 \\
Orientação Ego & $3.63( \pm .50)$ & $3.85( \pm .45)$ & $3.63( \pm .74)$ & $3.98( \pm .44)$ & .069 \\
\hline
\end{tabular}

Quadro 5

Comparação e diferenciação das orientações cognitivas em função da posição competitiva

\begin{tabular}{cccccccc}
\hline GRe & $\mathrm{C}$ & $\mathrm{L}$ & $\mathrm{P}$ & Piv & $\mathrm{U}$ & \\
& $(n=9)$ & $(n=10)$ & $(n=11)$ & $(n=11)$ & $(n=10)$ & $(n=6)$ & $\eta_{p}{ }^{2}$ \\
\hline $\mathrm{M}(\mathrm{DP})$ & $\mathrm{M}(\mathrm{DP})$ & $\mathrm{M}(\mathrm{DP})$ & $\mathrm{M}(\mathrm{DP})$ & $\mathrm{M}(\mathrm{DP})$ & $\mathrm{M}(\mathrm{DP})$ & \\
\hline Orientação Tarefa & $4.05( \pm .36)$ & $3.87( \pm .29)$ & $4.03( \pm .33)$ & $4.13( \pm .41)$ & $3.99( \pm .50)$ & $4.17( \pm .31)$ & .064 \\
Orientação Ego & $3.72( \pm .62)$ & $3.63( \pm .31)$ & $3.77( \pm .59)$ & $4.05( \pm .49)$ & $3.80( \pm .53)$ & $3.61( \pm .17)$ & .087 \\
\hline Gre = Guarda redes; $\mathrm{C}=$ Central; $\mathrm{L}=$ lateral; P = Ponta; Piv. = Pivot; U = Universal
\end{tabular}




\section{DISCUSSÃO}

$\mathrm{Na}$ nossa investigação, os andebolistas revelaram uma maior orientação para a tarefa do que para o ego, tal como nos estudos de Almeida (2009) e Vasconcelos-Raposo e Mahl (2005). Acreditamos que isto ocorre pelo facto do estudo se ter realizado numa modalidade coletiva o que leva a que, habitualmente, os jogadores sejam incentivados a dirigir preferencialmente os seus esforços e a sua prestação em função da equipa ao invés de valorizarem em demasia as suas metas pessoais.

A elevada orientação para a tarefa por parte dos atletas em estudo resulta dos feedbacks que estes recebem de todos aqueles que intervêm na sua prática desportiva. Quando um treinador dá feedbacks positivos aos atletas após a correta execução de uma tarefa e critica quando executam de forma errada o exercício, o treinador está a optar por um sistema de reforços. Os atletas irão manter os comportamentos que forem reforçados, isto é, se o treinador elogia a execução de um exercício, o atleta tenderá a manter a conduta.

Vejamos um exemplo da aplicação de reforço para a mestria, se um guarda-redes obtém 100\% de eficácia de defesa numa série de 10 remates e o treinador corrigir a sua postura, a posição em relação à baliza, o timing de saída à bola, o posicionamento dos braços e das pernas, etc., significa que está preocupado com a correta execução da tarefa por parte do atleta e não com o resultado. Isto quer dizer que o treinador valoriza a orientação para a mestria, preocupando-se com a melhoria das habilidades do atleta numa dada tarefa ao invés da preocupação com o resultado.

No que concerne ao escalão competitivo, os iniciados apresentam médias mais elevadas tanto na orientação cognitiva para o ego como na orientação cognitiva para a tarefa, seguidos dos jogadores seniores. Os nossos resultados vão de encontro ao estudo de Libório (2007) que encontrou valores superiores na orientação para o ego nos escalões inferiores, e ao estudo de Coroa (2009) que verificou que atletas muito novos se orientam preferencialmente para a tarefa. Verificaram-se apenas diferenças significativas na orientação para a tarefa entre o escalão de seniores e o escalão de juvenis, sendo que os primeiros apresentam médias mais altas. Os juvenis e juniores apresentam médias mais baixas em ambas as orientações cognitivas.

Os nossos resultados podem ser explicados pelo facto dos jogadores iniciados serem muito jovens, com idades compreendidas entre os $14 \mathrm{e}$ 15 anos, e ainda não terem adquirido uma clara definição e perceção dos conceitos abordados nos questionários. Em idades mais tenras, é habitual os atletas responderem às perguntas da forma que consideram ser mais positiva e correta, muitas vezes não correspondendo exatamente à realidade daquilo que vivenciam em relação à orientação das suas motivações. Tal como afirma Vygotsky (1994), nos primeiros estágios de desenvolvimento do jogo, a definição funcional dos conceitos e dos objetos ainda não foi atingida e as palavras ainda não se tornaram em algo concreto. Em idade mais avançadas, a compreensão de conceitos mais complexos vai aumentando e verifica-se assim um decréscimo de ambas as orientações cognitivas. Acreditamos que os atletas mais velhos respondem aos questionários com mais consciência e realismo que os atletas mais jovens. Elkonin (1998) defende que o jogo possibilita às crianças transcender tendências imediatas, enraizadas em motivações mais biológicas e primitivas, para seguir as regras sociais e atuar espelhando-se na realidade.

No escalão sénior verifica-se um aumento de ambas as orientações cognitivas que pode ser explicado com a profissionalização destes atletas. Com a profissionalização da atividade desportiva acresce-se, não só o envolvimento do atleta na modalidade que pratica, como também a responsabilidade para com a equipa onde se insere. Exige-se ao atleta que, não só execute as tarefas da maneria mais correta 
possível (orientação para a tarefa), mas também que os resultados apareçam (orientação para o ego), uma vez que o caracter competitivo está inerente à profissionalização de uma modalidade. Os feedbacks dados aos atletas acerca dos seus desempenhos na fase de aprendizagem (juvenis/juniores) têm um impacto evidente nas orientações cognitivas. Um feedback autorreferenciado vincula-se a uma orientação maior à tarefa, feedbacks socialmente comparados estão relacionados com uma maior orientação para o ego (Shih \& Alexander, 2000). A estabilização das médias das orientações cognitivas verificada no escalão sénior pode ser sustentada pela interação entre o desenvolvimento de crenças autorreferenciadas e a promoção de experiências em busca do melhor resultado, o que, segundo Bandura (1997), tem uma influência na ação, motivação e nos processos cognitivos.

Quanto aos anos de prática da modalidade, os nossos resultados vão de encontro ao do estudo de Rodrigues, Lázaro, Fernandes e Vasconcelos-Raposo (2009) onde verificaram que a orientação para a tarefa é superior nos atletas com mais anos de experiência. Santos (2009) também verificou no seu estudo que à medida que aumentam os anos de prática desportiva, aumenta a orientação motivacional para a tarefa. Verificou-se que os atletas mais experientes (grupo 4) apresentaram índices mais elevados tanto na orientação para o ego como na orientação para a tarefa, seguidos dos atletas do grupo 1. Aproximadamente na altura em que os atletas passam do grupo 1 para o grupo 2 e começam a adquirir mais vivências desportivas ocorre um período de descentralização cognitivo, por esse facto o grupo 2 é aquele que apresenta médias mais baixas relativamente às orientações cognitivas. Ao longo dos tempos, vários autores (Piaget, 1982; Elkonin, 1998) destacaram a importância do jogo para a superação do egocentrismo cognitivo e emocional, defendem que, através dele, a criança começa a reconhecer outros pontos de vista, a colocar-se no lugar do outro, perceber melhor o mundo que as rodeia e a estabelecer de forma mais eficaz inter-relações. Este conjunto de mudanças permite que se constituam novas operações intelectuais e que o pensamento atinja um nível mais elevado.

Posteriormente, com o aumento da experiência desportiva, é importante conceder aos atletas uma grande variedade de estímulos e vivências desportiva, bem como valorizar as aprendizagens técnicas e táticas. A estimulação das capacidades dos atletas permitirá a importante aquisição da motricidade fina e a possibilidade de um novo acréscimo das médias das orientações cognitivas. Ré (2011) acredita que o desenvolvimento em fases posteriores é influenciado pelo processo de aquisição de habilidades e capacidades motoras, o desempenho desportivo e a qualidade e quantidade dos estímulos vivenciados em fases iniciais. O mesmo autor defende ainda que na adolescência o atleta deve já possuir um padrão coordenativo e cognitivo, para que seja implementado o treino da força, velocidade e resistência que conduzam posteriormente à especialização de um atleta numa determinada modalidade.

Numa fase mais avançada, quando os jogadores já detêm de uma elevada experiência desportiva, as médias de ambas as orientações cognitivas tendem a aproximar-se devido à estabilização característica dos atletas profissionais. Para jogadores com vários anos de experiência é tão importante executar bem uma tarefa (orientação para a tarefa), como obter o melhor resultado numa dada competição (orientação para o ego), características resultantes da profissionalização enquanto atleta.

Não foram encontrados outros estudos que tivessem correlacionado o tempo de presença no clube com as orientações cognitivas dos atletas e por esse motivo não podemos comparar os resultados obtidos nesta investigação. Em todos os grupos a orientação para a tarefa está mais desenvolvida que a orientação para o ego, não se evidenciando diferenças 
significativas entre eles. No entanto, a orientação para a tarefa encontra-se mais desenvolvida nos grupos 2 e 4 . Isto pode ser explicado pelo facto de inicialmente quando os atletas integram o clube ainda não têm uma clara definição das suas orientações motivacionais. Com o aumento do tempo de presença no clube, estes vão valorizando mais a orientação para a tarefa uma vez que na fase de aprendizagem (camadas jovens) vão recebendo feedbacks no sentido da autorreferenciação e melhoria da execução de uma tarefa ao invés da valorização do resultado. Contudo, quando já se encontram no clube há algum tempo, além de lhes ser solicitado que sejam perfeccionistas nas suas prestações, também esperam que eles obtenham os melhores resultados nas competições disputadas. O clube estudado tem como preocupação, não só que os atletas evoluam técnica e taticamente, mas tem também como lema entrar numa competição sempre para vencer. Deste modo, o grupo com mais de 7 anos de permanência foram os que obtiveram não só médias mais altas na orientação para a tarefa como obtiveram, também, as médias mais altas na orientação para o ego.

Cada clube tem uma filosofia e ideais que vão sendo transmitidos aos atletas e outros agentes desportivos que dele fazem parte. A filosofia do clube envolve regras desportivas e sociais que vão sendo adquiridas e interiorizadas pelos atletas à medida que vai aumentando o seu tempo de presença no clube. Com o tempo, o ser humano aprende a usar racionalmente as suas capacidades, o ambiente torna-se interiorizado e o comportamento torna-se social e cultural (Vasconcelos-Raposo, 1993).

No que diz respeito à posição competitiva também não foram encontradas diferenças significativas ao nível das orientações cognitivas, tal como verificou Vasconcelos-Raposo e Mahl (2005). Destaca-se apenas os pontas que evidenciaram uma média mais alta na orientação para o ego e os jogadores universais que apresentaram uma média mais alta na orientação para a tarefa. Lázaro, Casimiro e Fernandes (2005) admitem a dificuldade de avaliar um jogador universal, contudo acreditam que este possa ser um atleta dotado de um conjunto de capacidades mentais que o fazem ser eficaz em qualquer posição. Os resultados obtidos pelo grupo dos pontas pode ser explicado pelo facto destes jogadores não serem tão participativos como um central ou um lateral na construção do jogo ofensivo, em que é de extrema importância executar corretamente todos os passes, organizarem o jogo e orientarem os colegas (tarefas mais dirigidas para a mestria), e também pelo facto dos pontas serem os principais intervenientes no contra-ataque, em que o principal objetivo é marcar golo (função mais relacionada com a orientação para o ego).

No entanto, existe uma certa dificuldade em avaliar o jogador de andebol em função da posição competitiva uma vez que o mesmo atleta pode ocupar várias posições no decorrer de um só jogo, ou seja, por vezes verifica-se uma falta de definição da posição do atleta (com exceção dos guarda-redes). Devido à imprevisibilidade e dinâmica do jogo de andebol, o mesmo atleta pode ocupar diferentes posições dependendo do desenho tático do treinador, da equipa estar a atacar ou defender, de estar a ganhar ou a perder, de se encontrar temporariamente sem um ou mais jogadores (devido às habituais exclusões de dois minutos), entre outras condições inesperadas.

\section{CONCLUSÕES}

No geral, os andebolistas abrangidos nesta investigação estão fortemente orientados para a tarefa e para o ego, apresentando, no entanto, níveis mais elevados de orientação para a tarefa, ou seja, além da elevada preocupação em executar corretamente uma determinada tarefa, têm também como objetivo a obtenção do melhor resultado numa dada competição.

Relativamente ao escalão competitivo e aos anos de prática da modalidade, inicialmente há 
uma diminuição das médias das orientações cognitivas devido à ocorrência da importante fase do descentralismo cognitivo e emocional incitada pelo próprio jogo. A participação num dado desporto tem um papel fundamental na capacidade do indivíduo em se colocar no lugar do outro e conduzir o pensamento para níveis mais elevados, sendo preponderante no início da adolescência e na iniciação da prática desportiva. Após esse período de adaptação, caracterizado por vivências desportivas, feedbacks e diversas aprendizagens, os atletas vão aumentando novamente as suas orientações cognitivas. Com a experiência tendem a convergir para uma estabilização, em que há uma aproximação entre as médias da orientação cognitiva para a tarefa e da orientação cognitiva para o ego.

Após um determinado tempo de presença num dado clube, os atletas vão adquirindo atitudes e seguindo orientações em conformidade com os feedbacks que vão recebendo. Cada clube tem uma filosofia e ideais característicos que vão transmitindo aos seus agentes desportivos e que vão, consequentemente, sendo interiorizados e assumidos por estes, os atletas evidenciam assim características do clube a que pertencem. Uma vez que o clube estudado os orienta preferencialmente para a correta execução dos exercícios e movimentos do andebol, valorizando igualmente a obtenção do melhor resultado, os atletas evidenciam níveis elevados de orientação cognitiva não só para a tarefa como também para o ego.

Há uma certa dificuldade em avaliar o jogador de andebol em função da posição competitiva, visto que muitas vezes os atletas precisam de estar preparados para assumir várias posições competitivas num mesmo jogo devido à dinâmica e imprevisibilidade do andebol.

De um modo geral, defendemos que em qualquer investigação desta área, o desportista deve ser visto e interpretado numa perspetiva holística, pois o atleta é o resultado de um conjunto complexo de fatores físicos, fisiológicos, sociais, culturais e psicológicos que inte- ragem entre si.

\section{Agradecimentos:}

Nada declarado.

Conflito de Interesses:

Nada declarado.

\section{Financiamento:}

Nada declarado.

\section{REFERÊNCIAS}

Almeida, J. (2009). Perfil de actividade e alterações fisiológicas e funcionais induzidas pelo jogo de andebol em jogadores de diferente nível competitivo. Dissertação de Mestrado, Universidade do Porto, Porto, Portugal.

Bandura, A. (1997). Self-eficacy: The exercise of control. New York: W.H. Freeman.

Buckworth, J., \& Dishman, R. (2007). Exercise adherence (pp. 509-536). In G. Tenenbaum, \& R. Eklund, Handbook of Sport Psychology. New York: Wiley \& Sons.

Coroa, S. (2009). Objectivos de realização, clima motivacional e ansiedade em jogadores de futebol: Um estudo realizado no escalão de escolas. Dissertação de Licenciatura, Faculdade de Desporto da Universidade do Porto, Porto, Portugal.

Cox, R. (2007). Sport Psychology: Concepts and applications (6 $6^{\text {th }}$ Ed.). New York: McGraw-Hill.

Elkonin, D. (1998). Psicologia do jogo. São Paulo: Martins Fontes.

Fernandes, H., \& Vasconcelos-Raposo, J. (2010). Análise factorial confirmatória do TEOSQp. Psicologia: Reflexão e Crítica, 23(1), 92-101.

Ferreira, R., Costa, V., Penna, E., Samulsky, D., \& Moraes, L. (2012). Habiliddes mentais de nadadores brasileiros de alto rendimento. Motricidade, 8(S2), 946-955. Doi: 10.6063/motricidade.8(0). 649

Lázaro, J., Casimiro, E., \& Fernandes, H. (2005). Determinação do perfil psicológico de prestação de andebol português: Um estudo em atletas da Liga e da Divisão de Elite. Obtido em Novembro de 2010, de psicologia.pt. O Portal dos Psicólogos: 
http://www.psicologia.com.pt/artigos/textos/ A0269.pdf

Libório, M. (2007). Negativismo competitivo e orientações motivacionais em atletas de orientação pedestre. Monografia, Universidade de Trás-os-Montes e Alto Douro, Vila Real, Portugal.

Piaget, J. (1982). O nascimento da inteligência da criança (4a Ed.). Rio de Janeiro: Zahar.

Ré, A. (2011). Crescimento, maturação e desenvolvimento na infância e na adolescência: implicações para o esporte. Motricidade, 7(3), 55-67.

Rodrigues, A., Lázaro, J., Fernandes, H., \& Vasconcelos-Raposo, J. (2009). Caracterização dos níveis de negativismo, activação, autoconfiança e orientações motivacionais de alpinistas. Motricidade, 5(2), 63-86.

Santos, A. (2009). A ansiedade e orientações motivacionais em praticantes de atletismo e basquetebol em cadeira de rodas e a sua relação com o rendimento desportivo. Monografia, Universidade de Trás-os-Montes e Alto Douro, Vila Real, Portugal.

Shih, S., \& Alexander, J. (2000). Interacting effects of goal setting and self or other-referenced feedback on children's development of self-efficacy and cognitive skill within the Taiwanese classroom. Journal of Educational Psychology, 92, 536-543.
Silva, M. (2006). Orientação motivacional e negativismo competitivo em praticantes de canoagem de elite. Monografia, Universidade de Trás-os-Montes e Alto Douro, Vila Real, Portugal.

Vasconcelos-Raposo, J. (1993). Os fatores bio-psico-socio-culturais que influenciam e determinam a busca pela excelência em atletas de elite portugueses. Tese de doutoramento. Universidade de Trás-os-Montes e Alto Douro.

Vasconcelos-Raposo, J., \& Mahl, A. (2005). Orientação cognitiva de atletas profissionais de Futebol do Brasil. Motricidade, 1(4), 253-265.

Vygotsky, L. (1994). A formação social da mente. São Paulo: Martins Fontes.

Weinberg, R., \& Gould, D. (2007). Foundations of Sport and Exercise Psychology (4th Ed.). Champaign: Human Kinetics. 